\title{
Encuesta sobre la elección de pareja a estudiantes de la Universitat Pompeu Fabra, Barcelona (20 a 27 años)
}

\author{
Survey about mate preferences by university students \\ of Universitat Pompeu Fabra (from 20 to 27 years old)
}

\author{
Carmen Maté ${ }^{1}$ \\ Nolasc Acarín \\ Agencia de Ecología Urbana, España
}

(Rec: Febrero 2011 - Acep: Mayo 2011)

\begin{abstract}
Resumen
Los estudios sobre la elección de pareja determinan si las características deseables y seleccionadas son universales, o bien si determinadas diferencias sexuales en la elección de pareja están influidas por la cultura como factor modulador, o anulador, de las preferencias evolutivas que pudieran existir. Por ello comparamos los resultados con otras encuestas realizadas en diferentes épocas y culturas. El objeto principal de este estudio es verificar si se mantienen algunas predicciones evolucionistas más evidentes sobre las diferencias sexuales en las preferencias de emparejamiento, diferenciando las relaciones estables de las puntuales. En las respuestas dadas por los universitarios, las características que se valoran para tener una pareja estable son el compromiso, la amabilidad, la sinceridad y la comprensión en general. Al comparar las preferencias en función del sexo, se observan diferencias estadísticamente significativas con respecto al atractivo físico, más valorado por los varones, y la posición social por las mujeres. Cuando se trata de emparejamientos ocasionales, el atractivo físico es la característica más deseada, después la simpatía y la amabilidad. Existen diferencias estadísticamente significativas entre lo que valoran ambos sexos en las relaciones puntuales; las mujeres, la simpatía y la posición social y los hombres, el atractivo físico y la personalidad enigmática.
\end{abstract}

Palabras clave: elección de pareja, variaciones en la elección de pareja, encuesta a universitarios.

\begin{abstract}
The election about mate preferences studies, determine if the characteristics desirable and selected are universal, or if the difference sexual preferences are influenced by the culture as a modulator factor in the evolutionary preferences that could exist. So that it's the reason we compared the results with other surveys made in different times and cultures. The main object the study goal is to verify if some more evident evolutionary predictions stay on the sexual differences in the stable mate relationships preferences differentiating the stable relations from the fleeting ones. The students answers show that, the characteristics that are valued to have a stable pair are the commitment, the amiability, sincerity and the understanding in general. When comparing the sex preferences based on sex, are observed statically significant differences with respect to the attractive physicist, more valued by the men, and the social position by the women. When they choose for a fleeting mate they selected physical attractiveness more than, later the affection and the amiability. There are statistically significant differences between which they value both sexes in the precise relations, the women, the affection and the social position and the men, the physical attractiveness and the enigmatic personality.
\end{abstract}

Key words: mate preferences, variation in the mate preferences, students survey.

${ }^{1}$ Correspondencia a: Carmen Maté. Dirección Postal: c/Escar nº1, 3º8039 Barcelona, España. E-mail: carmenmate@bcncologia. net. 


\section{Introducción}

Una parte de la encuesta realizada a los estudiantes de Humanidades de la Universitat Pompeu Fabra (UPF) en Barcelona se inspiró en los estudios realizados por Buss $(1984,1989)$, Buss et al. (1990) y Buss y Schmitt (1993), sobre emparejamientos permanentes y temporales en los que se valoran las características que hombres y mujeres buscan según formen parejas temporales o permanentes. La encuesta constaba de 42 preguntas que se agrupan en cuatro bloques temáticos, el primer trabajo más genérico trataba sobre las relaciones sexuales (Maté y Acarín, 2010). Otras preguntas versaban sobre el festejo y la seducción (Maté y Acarín, en preparación) y por último sobre la formación de pareja y la familia. Este segundo artículo muestra los valores que se seleccionan en una pareja para tener una relación estable frente a una fugaz. Este aspecto de la conducta sexual fue estudiado a partir de seis preguntas. El formato de las mismas consistía en una selección de diferentes valores y características que además implicaba priorizar las opciones seleccionadas, otras preguntas tenían respuestas dicotómicas. En las preguntas que debían seleccionar y priorizar los valores, a partir de una lista de once, las características fueron similares a los propuestos en los estudios de Buss $(1984,1989,1990,1993)$ y los publicados por Demoscopia (1997).

La mayor parte de los datos disponibles acerca de las actitudes y los comportamientos sexuales provienen de las investigaciones de EEUU y otras culturas, estudios realizados a través de encuestas basadas en cuestionarios o entrevistas. Estos trabajos permiten mostrar la diversidad de expresión humana y mejorar la comprensión de las normas y valores más universales.

En los estudios sobre la elección de pareja se determinan si las características deseables y seleccionadas son universales, es decir, las compartimos todos los humanos, o bien si determinadas diferencias sexuales en la elección de pareja están influidas por la cultura como factor modulador, o anulador, de las preferencias evolutivas que pudieran existir. Por ello compararemos los resultados con los obtenidos en otras culturas presentados en la encuesta realizada por Buss (1989; Buss et al. 1990 y Buss et al. 1993). En 37 culturas localizadas en 6 continentes y 5 islas de Australia a Zambia, la edad de la población estudiada oscilaba de 14 a 70 años (n=10.047). Este estudio documenta las preferencias sexuales universales que manifiestan hombres y mujeres por determinadas características en un compañero y exploran los cambios que tienen lugar cuando el objetivo pasa de ser una relación sexual fortuita, al compromiso en una relación estable. También comparemos nuestros resultados con los de Ford y Beach (1951), que analizaron la sexualidad humana en 190 sociedades y es la base del trabajo de referencia sobre estudios de sexualidad elaborado por Laumann, Gagnon, Michael, Michaels (1994), que establece las bases de la encuesta nacional en EEUU.

La elección de pareja es una estrategia sexual que se ajusta a un problema adaptativo específico, cómo identificar al compañero/a deseable, o superar a los competidores a la hora de atraerlo. Además, por debajo de la estrategia sexual hay mecanismos psicológicos como las preferencias por una pareja concreta, los sentimientos amorosos, el deseo sexual o los celos (Buss, 1994). Estos mecanismos son sensibles a la información o a las señales del mundo exterior; como los rasgos físicos, los signos de interés sexual o las indicaciones de infidelidad potencial. Nuestros mecanismos psicológicos son también sensibles a la información sobre nosotros mismos, como la capacidad de atraer a una pareja que pueda ser deseable.

El objeto principal de este estudio es verificar si se mantienen algunas predicciones evolucionistas más evidentes sobre las diferencias sexuales en las preferencias de emparejamiento, por ejemplo si los hombres buscan la juventud y el atractivo físico y las mujeres una buena posición y seguridad económica. También se pretende mostrar las diferencias en la elección de valores, cuando se busca deliberadamente una relación corta, puntual o fugaz, una unión temporal o una breve aventura, y cuando lo hacen, sus preferencias cambian.

Además, con el mismo formato de pregunta que en el cuestionario servía para preguntar sobre los caracteres que elegiría en su pareja, se formularon un par de preguntas similares para que indicaran aquellas características que valoraban en sus progenitores y si pensaban que el modelo de pareja de sus padres les había influido.

\section{Método}

\section{Participantes}

La población estudiada son los estudiantes de Humanidades de la Universitat Pompeu Fabra (UPF) con una media de edad de 22 años -el mínimo de 19 y el máximo de 41-, el 96,2\% de la muestra está comprendida entre 19 a 27 años. La 
encuesta se inició en el curso 1999-2000 y finalizó el 2003-2004 participando un total de 838 alumnos (253 varones y 585 mujeres). Esta proporción es la misma que se observa en los estudiantes, siendo dos terceras partes mujeres. Todos los participantes de la encuesta eran voluntarios y anónimos y estaban matriculados en tercero y cuarto curso de la licenciatura de Humanidades. El universo fue el conjunto de estudiantes de Humanidades que se matriculaban a las asignaturas impartidas por los autores (240 estudiantes/año); la totalidad de alumnos de los cursos de tercero y cuarto era de 1200. La encuesta se realizaba en un día de clase y en cada formulario el estudiante solo indicaba la edad, el sexo y la fecha de la realización. Al recoger los formularios se les identificaba con un número para el control de la muestra y para garantizar el anonimato.

\section{Instrumentos}

Los autores elaboraron una encuesta de 42 preguntas sobre las experiencias y actitudes sexuales. En concreto, en este artículo se analizan las relacionadas con los valores que se seleccionan en una pareja para tener una relación estable frente a una fugaz. Este aspecto de la conducta sexual fue estudiado a partir de seis preguntas. El formato de las mismas consistía en una selección de diferentes valores y características que además implicaba priorizar las opciones seleccionadas, mientras otras preguntas tenían respuestas dicotómicas. En las preguntas que debían seleccionar y priorizar los valores, a partir de una lista de once, las características fueron similares a las propuestas en la bibliografía.

\section{Procedimiento}

El error de la muestra, en el supuesto de una selección aleatoria, para un nivel de confianza $95 \%$ $(2 \sigma)$ es de $0,01(1 \%)$ para el conjunto de la muestra, siendo $\mathrm{N}=1200$, que era la totalidad de alumnos en los cursos de tercero-cuarto durante el período de estudio.

Para el análisis confirmatorio se igualaron las muestras para hombres y mujeres $\mathrm{N}=500$ (250 varones y 250 mujeres) para comprobar si existían diferencias en las respuestas en función del sexo. La muestra de las mujeres se seleccionó al azar con una opción que tiene el programa de análisis estadístico SPSS.

\section{Análisis de los datos}

Las pruebas estadísticas empleadas para las variables cualitativas son la tablas de contingencia con el cálculo de la $\chi^{2}$ de Pearson y los residuos ajustados (empleando como referencia el valor estadístico de prueba 1,96 en valor absoluto para una distribución normal y un nivel de significación de 0,05$)$ de Haberman, 1978. En los casos que los valores esperados de al menos el $80 \%$ de las celdas de la tabla de contingencia eran inferiores a 5 , se utilizó la prueba exacta de Fisher.

\section{Resultados}

Las once características que debían elegir y priorizar eran: la sinceridad, la inteligencia, la simpatía (o buen humor), la comprensión, las atenciones (amabilidad, afecto), la fidelidad, el atractivo físico, inspirar confianza, el dinero (buena posición social),

Tabla 1. Caracteres significativamente más valorados por hombres y mujeres en la elección de una pareja estable.

\begin{tabular}{llll}
\hline Caracteres más valorados en una pareja estable & & & \\
& Prueba estadística & gl & Valor $\mathrm{p}$ \\
\hline La sinceridad & $12,44^{\mathrm{a}}$ & 4 & $\mathrm{p}<0,05^{*}$ \\
La inteligencia & $12,25^{\mathrm{a}}$ & 4 & $\mathrm{p}<0,05^{*}$ \\
Simpatía, buen humor & $10,79^{\mathrm{a}}$ & 4 & $\mathrm{p}<0,05^{*}$ \\
Comprensión & $10,35^{\mathrm{a}}$ & 4 & $\mathrm{p}<0,05^{*}$ \\
Atenciones, amabilidad & $6,39^{\mathrm{a}}$ & 4 & $\mathrm{~ns}$ \\
Fidelidad & $18,71^{\mathrm{a}}$ & 4 & $\mathrm{p}<0,05^{*}$ \\
Atractivo Físico & $42,98^{\mathrm{a}}$ & 4 & $\mathrm{P}<0,001^{* *}$ \\
Confianza & $17,2^{\mathrm{a}}$ & 4 & $\mathrm{p}<0,05^{*}$ \\
Dinero, posición social & $7,40^{\mathrm{b}}$ & & $\mathrm{p}<0,05^{*}$ \\
Capacidad de iniciativa & $5,38^{\mathrm{b}}$ & $\mathrm{ns}$ \\
Personalidad enigmática & $8,73^{\mathrm{b}}$ & & $\mathrm{p}<0,05^{*}$ \\
\hline${ }^{\mathrm{a}}$ Chi-cuadrado & & & \\
${ }^{\mathrm{b}}$ F de Fisher & & &
\end{tabular}


Tabla 2. Características que debería tener la pareja significativamente más valoradas por hombres y mujeres.

\begin{tabular}{lccc}
\hline & \multicolumn{2}{c}{ Características que debería tener la pareja } \\
& Prueba Estadística & $\mathrm{gl}$ & Valor $\mathrm{p}$ \\
\hline La sinceridad & $4,32^{\mathrm{a}}$ & 4 & $\mathrm{~ns}$ \\
La inteligencia & $19,16^{\mathrm{a}}$ & 4 & $\mathrm{p}<0,05^{*}$ \\
Simpatía, buen humor & $3,20^{\mathrm{a}}$ & 4 & $\mathrm{~ns}$ \\
Comprensión & $29,28^{\mathrm{a}}$ & 4 & $\mathrm{p}<0,001^{* *}$ \\
Atenciones, amabilidad & $5,01^{\mathrm{a}}$ & 4 & $\mathrm{~ns}$ \\
Fidelidad & $26,37^{\mathrm{a}}$ & 4 & $\mathrm{p}<0,001^{* *}$ \\
Atractivo Físico & $60,71^{\mathrm{a}}$ & 4 & $\mathrm{p}<0,001^{* *}$ \\
Confianza & $10,10^{\mathrm{a}}$ & 4 & $\mathrm{p}<0,05^{*}$ \\
Dinero, posición social & $6,87^{\mathrm{b}}$ & & $\mathrm{p}<0,05^{*}$ \\
Capacidad de iniciativa & $7,97^{\mathrm{a}}$ & 3 & $\mathrm{p}<0,05^{*}$ \\
Personalidad enigmática & $6,70^{\mathrm{b}}$ & & $\mathrm{ns}$ \\
\hline
\end{tabular}

${ }^{\text {a }}$ Chi-cuadrado

${ }^{\mathrm{b}} \mathrm{F}$ de Fisher

la capacidad de iniciativa, y por último la personalidad enigmática o misteriosa. Estas características personales aparecen en todas las preguntas en las que tenían que seleccionar y priorizar las deseadas en una pareja.

\section{Elección de pareja estable}

El 43,4\% (n=364) seleccionó la sinceridad en primer lugar, el 16,8\% ( $\mathrm{n}=141)$ marcaba la inteligencia en segundo lugar, la tercera característica con un $17,1 \%(n=143)$ fue la amabilidad y como cuarta con un $14,7 \%(n=123)$ la comprensión.

Se obtienen diferencias estadísticamente significativas entre hombres y mujeres en las características que más valoran en la pareja cuando la relación es permanente, en la tabla 1 se muestran los resultados. Así, las mujeres valoran más que los hombres la sinceridad y los hombres más la inteligencia, la confianza, la simpatía. Las mujeres también valoran, más que los hombres, la comprensión y la fidelidad. Respecto al atractivo físico, es más importante para los hombres cuando la relación es estable así como también valoran más una personalidad enigmática o misteriosa. Las mujeres otorgan más relevancia que los hombres al dinero y la posición social.

No se observaron diferencias estadísticamente significativas entre hombres y mujeres en la

Tabla 3. Caracteres significativamente más valorados por hombres y mujeres para mantener una relación fugaz.

\begin{tabular}{lccc}
\hline & Caracteres más valorados en una pareja fugaz & \\
& Prueba Estadística & $\mathrm{gl}$ & Valor $\mathrm{p}$ \\
\hline La sinceridad & $2,84^{\mathrm{b}}$ & $\mathrm{Ns}$ \\
La inteligencia & $0,65^{\mathrm{b}}$ & 4 & $\mathrm{Ns}$ \\
Simpatía, buen humor & $41,81^{\mathrm{a}}$ & 4 & $\mathrm{p}<0,001^{* *}$ \\
Comprensión & $3,22^{\mathrm{b}}$ & 4 & $\mathrm{Ns}$ \\
Atenciones, amabilidad & $4,95^{\mathrm{a}}$ & 4 & $\mathrm{Ns}$ \\
Fidelidad & $2,44^{\mathrm{b}}$ & 4 & $\mathrm{Ns}$ \\
Atractivo Físico & $45,65^{\mathrm{a}}$ & 4 & $\mathrm{P}<0,001^{* *}$ \\
Confianza & $8,03^{\mathrm{a}}$ & 4 & $\mathrm{Ns}$ \\
Dinero, posición social & $16,62^{\mathrm{b}}$ & 4 & $\mathrm{p}<0,001^{* *}$ \\
Capacidad de iniciativa & $9,89^{\mathrm{b}}$ & 4 & $\mathrm{p}<0,05^{*}$ \\
Personalidad enigmática & $25,04^{\mathrm{a}}$ & 4 & $\mathrm{p}<0,001^{* *}$ \\
\hline
\end{tabular}

${ }^{\mathrm{a}}$ Chi-cuadrado

${ }^{\mathrm{b}} \mathrm{F}$ de Fisher 
selección de las características como las atenciones, la amabilidad y el afecto, ni en la capacidad de iniciativa.

En el cuestionario, después de las tres primeras preguntas se formula de nuevo otra, para preguntar qué características debería tener una persona para que sea elegida como su pareja. La sinceridad vuelve a ser la primera característica más seleccionada con $43,8 \%(\mathrm{n}=367)$, la segunda es la inteligencia con $20,3 \%(n=170)$, la tercera con $16,2 \%(n=136)$ es la simpatía y la cuarta es la amabilidad con un $16,2 \%(n=136)$. Solo se detecta una discrepancia en la tercera característica seleccionada cuando se pregunta por una posible pareja se opta por la simpatía en vez de la amabilidad.

No se observan diferencias estadísticamente considerables en las respuestas de hombres y mujeres respecto a las características siguientes: sinceridad, simpatía, amabilidad y atenciones, y personalidad enigmática-misteriosa. Los hombres valoran más la inteligencia y el atractivo físico. Las mujeres dan más importancia a la comprensión, la fidelidad, el dinero y la posición social y por último la capacidad de iniciativa (Tabla 2).

Tabla 4. Caracteres significativamente más valorados en el padre por hombres y mujeres.

\begin{tabular}{|c|c|c|c|}
\hline \multicolumn{4}{|c|}{ Características que más agradan del padre } \\
\hline & Prueba Estadística & gl & Valor $\mathrm{p}$ \\
\hline La sinceridad & $11,64^{\mathrm{a}}$ & 4 & $\mathrm{p}<0,05^{*}$ \\
\hline La inteligencia & $5,17^{\mathrm{a}}$ & 4 & ns \\
\hline Simpatía, buen humor & $6,31^{\mathrm{a}}$ & 4 & ns \\
\hline Comprensión & $12,59^{\mathrm{a}}$ & 4 & $\mathrm{p}<0,05^{*}$ \\
\hline Atenciones, amabilidad & $5,52^{\mathrm{a}}$ & 4 & ns \\
\hline Fidelidad & $3,57^{\mathrm{a}}$ & 4 & $\mathrm{~ns}$ \\
\hline Atractivo Físico & $3,29^{\mathrm{a}}$ & 4 & ns \\
\hline Confianza & $3,63^{\mathrm{a}}$ & 4 & ns \\
\hline Dinero, posición social & $4,08^{b}$ & & ns \\
\hline Capacidad de iniciativa & $8,89^{\mathrm{a}}$ & 4 & ns \\
\hline Personalidad enigmática & $11,25^{\mathrm{a}}$ & 4 & $\mathrm{p}<0,05^{*}$ \\
\hline
\end{tabular}

${ }^{a}$ Chi-cuadrado

${ }^{\mathrm{b}} \mathrm{F}$ de Fisher

Figura 1. Las diferencias entre los hombres y las mujeres en las respuestas sobre la influencia del modelo de pareja de los padres en su relación.

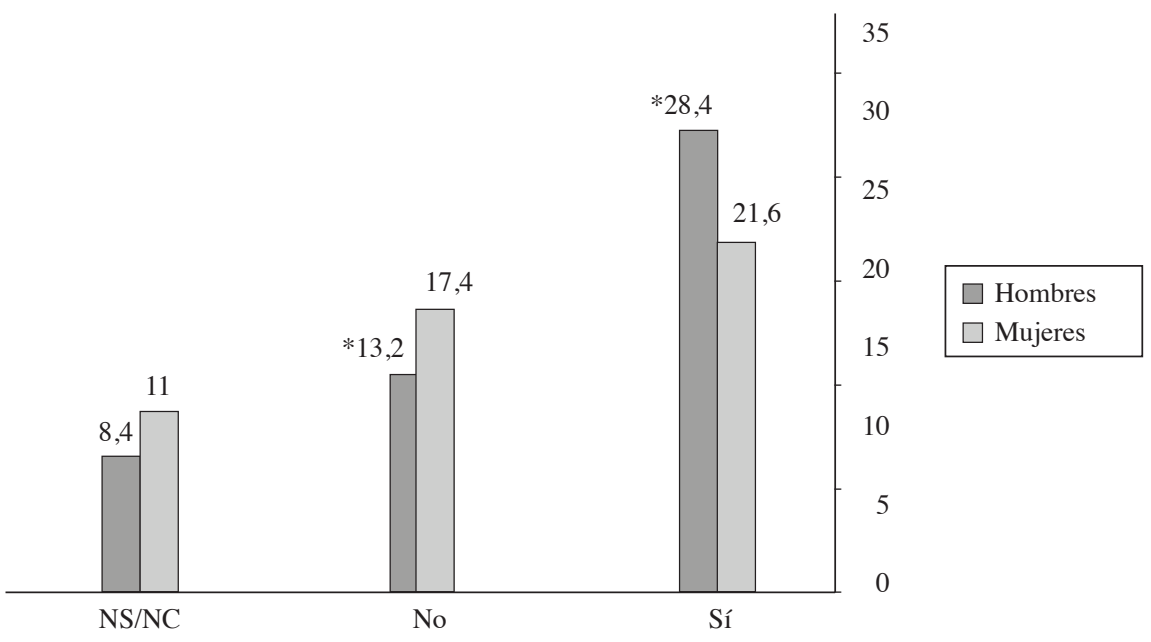


Tabla 5. Caracteres significativamente más valorados en la madre por hombres y mujeres.

\begin{tabular}{lccc}
\hline & \multicolumn{2}{c}{ Características que más agradan de la madre. } & \\
& Prueba Estadística & gl & Valor $\mathrm{p}$ \\
\hline La sinceridad & $1,30^{\mathrm{a}}$ & 4 & $\mathrm{~ns}$ \\
La inteligencia & $6^{\mathrm{a}}$ & 4 & $\mathrm{~ns}$ \\
Simpatía, buen humor & $7,12^{\mathrm{a}}$ & 4 & $\mathrm{~ns}$ \\
Comprensión & $5,02^{\mathrm{a}}$ & 4 & $\mathrm{~ns}$ \\
Atenciones, amabilidad & $5,14^{\mathrm{a}}$ & 4 & $\mathrm{~ns}$ \\
Fidelidad & $10,25^{\mathrm{a}}$ & 4 & $\mathrm{p}<0,05^{*}$ \\
Atractivo Físico & $3,46^{\mathrm{b}}$ & 4 & $\mathrm{~ns}$ \\
Confianza & $9,06^{\mathrm{a}}$ & 4 & $\mathrm{~ns}$ \\
Dinero, posición social & $4,39^{\mathrm{b}}$ & & $\mathrm{ns}$ \\
Capacidad de iniciativa & $7,76^{\mathrm{a}}$ & 5 & $\mathrm{~ns}$ \\
Personalidad enigmática & $2,54^{\mathrm{b}}$ & & $\mathrm{ns}$ \\
\hline
\end{tabular}

${ }^{\text {a }}$ Chi-cuadrado

${ }^{\mathrm{b}} \mathrm{F}$ de Fisher

\section{Elección para una pareja fugaz}

Cuando se trata de seleccionar y priorizar las mismas once características en una pareja para una relación corta, el 41,3\% (n=346) marca como primera el atractivo físico, la segunda con $24,2 \%$ $(\mathrm{n}=203)$ la simpatía, en tercer lugar con $17,9 \%$ $(\mathrm{n}=150)$ la amabilidad y la cuarta con $11,3 \%(\mathrm{n}=95)$ la personalidad enigmática o misteriosa.

Los resultados del análisis de las diferencias en las respuestas entre hombres y mujeres se muestran en la tabla 3. No se observan diferencias estadísticamente significativas entre hombres y mujeres en las respuestas respecto a las características siguientes: sinceridad, inteligencia, comprensión, amabilidad y atenciones, fidelidad, confianza. En cambio, la simpatía es más valorada por las mujeres que por los hombres en las relaciones puntuales así como valoran también más el dinero y la posición social y la capacidad de iniciativa. Los hombres, al contrario, valoran más el atractivo físico y la personalidad enigmática, misteriosa.

\section{Influencia de sus progenitores en el modelo de pareja}

El 54,2\% (n=454) piensa que el modelo de relación de sus padres ha influido en sus relaciones de pareja, el 28,9\% ( $\mathrm{n}=240)$ piensa que no, un $16,2 \%$ $(\mathrm{n}=134)$ no sabe y $1,4 \%$ no contesta.

Se obtienen diferencias estadísticamente notorias entre hombres y mujeres respecto a la influencia del modelo de pareja de sus progenitores $(\mathrm{F}=9,86$; $\left.\mathrm{p}<0,05^{*}\right)$, siendo las mujeres las que responden afirmativamente más (Fig. 1).

La encuesta acababa con dos preguntas semejantes a las analizadas anteriormente, de entre las mismas once características tenían que escoger y priorizar cuatro que más les agradaran de su padre. La primera escogida es la inteligencia con 19,9\% $(n=167)$, la segunda con $14 \%(n=117)$ la comprensión, la tercera con $15,5 \%(\mathrm{n}=130)$ las atenciones, amabilidad, afecto y la cuarta con $14,1 \%(n=118)$ la simpatía y el buen humor.

En la tabla 4 se muestran las diferencias estadísticamente significativas entre hombres y mujeres respecto a las características que más agradan del padre. Los hombres marcan más la sinceridad y la comprensión. Las mujeres resaltan más la personalidad enigmática.

La última pregunta del formulario era idéntica a la anterior, pero referida a la madre. El 28,5\% $(n=239)$ selecciona la comprensión, en segundo lugar con un $23 \%(\mathrm{n}=193)$ las atenciones, la amabilidad, el afecto, la tercera con $13,1 \%(n=110)$ es la sinceridad y la cuarta la simpatía y el buen humor con $16 \%(\mathrm{n}=134)$.

En la tabla 5 se muestran las diferencias estadísticamente relevantes en las repuestas de hombres y mujeres respecto a la fidelidad, siendo las mujeres quienes valoran más esta característica en su madre. 


\section{Discusión}

En los años cincuenta, Langhorne y Secord (1955) estudiaron las respuestas que 5000 estudiantes universitarios dieron cuando tenían que identificar las características que deseaban en una futura pareja. Los hombres mencionaron el atractivo físico mucho más que ellas. Las mujeres atribuyen el mismo grado de importancia a la posición social, el prestigio, el rango, el poder y la reputación, y lo hacen con mucha mayor frecuencia que los hombres.

En otro estudio transgeneracional sobre la pareja realizado en EEUU a lo largo de 50 años, de 1939 a 1989, se midió el valor que los hombres y las mujeres concedían a diferentes características, en intervalos de una década, para determinar si cambiaban con el tiempo las preferencias de emparejamiento. En todos los casos, los hombres consideran más importante y deseable que las mujeres, el atractivo físico y la belleza. Las mujeres, en cambio, lo valoraban como no muy importante (Buss 1985; Hudson \& Henze, 1969; Buss \& Barnes 1986).

En el estudio internacional realizado por Buss et al. $(1989,1990)$, en 37 culturas sobre la elección de pareja-con independencia del país, el hábitat, el sistema de matrimonio o las condiciones de vidalos hombres valoran la apariencia física más que las mujeres. Nuestros datos corroboran los obtenidos en los trabajos mencionados con anterioridad y apuntan a que la preferencia masculina por compañeras físicamente atractivas podría ser un mecanismo psicológico de la especie que transmite la cultura.

Aunque los universitarios estudiados aprecian especialmente la sinceridad, la inteligencia y la simpatía por encima del atractivo físico, también se obtienen diferencias estadísticamente significativas respecto a la valoración del dinero y la posición social, siendo las mujeres quienes muestran más preferencia por éstos. Los resultados de la encuesta de Demoscopia (1997) muestran un perfil similar a los nuestros en las respuestas genéricas (teniendo en cuenta toda la muestra).

Otros estudios sistemáticos efectuados a partir de los anuncios clasificados de los diarios -con una muestra de 1111 anuncios- muestran que las mujeres valoran, mucho más que los hombres, los recursos económicos y que la sinceridad era la cualidad preferida por las féminas (Wiederman \& Allgeier, 1992, 1993). Por tanto, las diferencias sexuales en la preferencia por los recursos no están limitadas a estudiantes universitarios, ni relacionadas con el método de la encuesta, tampoco están restringidos ni a EEUU, ni a las sociedades occidentales, pues el estudio internacional sobre la elección de pareja (Buss 1989, 1990) llevado a cabo durante cinco años (1984-89) en 37 culturas de 6 continentes y 5 islas ha confirmado la universalidad de estas preferencias. La muestra fue de 10.047 personas, los participantes procedían desde países que practicaban la poligamia a otros donde la norma era la monogamia. Los participantes valoraban 18 características en una pareja o cónyuge potenciales, en una escala que iba de sin importancia a indispensable. Las mujeres concedieron más trascendencia que los hombres a un buen porvenir económico, valorando los recursos económicos un $100 \%$ más que los hombres o aproximadamente el doble.

La diferencia sexual permanece invariable, las mujeres desean que la pareja tenga recursos económicos en mayor medida que los hombres. En otro estudio de 186 sociedades que abarcaba desde los pigmeos Mbuji de África a los esquimales aleut, los hombres de posición social más elevada tenían más bienes y mejores alimentos para sus hijos y esposas (Betzig, 1986).

En las respuestas dadas por los universitarios estudiados, las características que se valoran para tener una pareja estable son el compromiso, la amabilidad, la sinceridad y la comprensión en general. En cambio, cuando se comparan las preferencias de hombres y mujeres se observan diferencias con respecto al atractivo físico que es más valorado por los varones, y las mujeres dan más importancia a la posición social. En un estudio realizado a partir de 800 anuncios por palabras, la sinceridad era la característica más buscada por las mujeres (Harrison \& Saeed, 1977). Así la sinceridad, la inteligencia, la amabilidad y los recursos proporcionan información importante sobre los beneficios que una posible pareja puede reportar.

En el estudio internacional sobre elección de pareja, las mujeres muestran una clara preferencia por compañeros amables y comprensivos. En $32 \mathrm{de}$ las 37 culturas, ambos sexos coinciden en su evaluación de la amabilidad de las tres cualidades -de más de 13-más importantes en la pareja. En nuestro estudio sobre los universitarios, también se obtienen resultados similares respecto a la amabilidad y la comprensión cuando se busca una pareja estable. Estos resultados son semejantes a los obtenidos por Demoscopia (1997), que realiza una encuesta entre universitarios en todo el ámbito nacional y en todas las áreas de conocimiento, con una muestra de 4000 estudiantes encuestados.

La amabilidad es una característica duradera de la personalidad que tiene muchos elementos, pero 
en el fondo se halla el compromiso de los recursos. La amabilidad indica la capacidad y disposición de un posible compañero a dedicar sus recursos. Pedir amor, sinceridad y amabilidad es una forma de asegurarse un compromiso respecto a los recursos.

Pruebas de docenas de estudios documentan que la mujer estadounidense moderna valora los recursos económicos en su pareja mucho más que el hombre (Buss, 1994) En un estudio realizado en 1939 se midieron 18 características de hombres y mujeres estadounidenses relacionadas con lo que desearían en la pareja o el cónyuge, valorándolas de irrelevantes a indispensables. Las mujeres no consideran las buenas perspectivas financieras como absolutamente indispensables, pero sí importantes. Los hombres las consideraron deseables, pero no muy importantes. En 1939 las mujeres valoraban el óptimas que los hombres unas buenas perspectivas financieras (Buss 1994), hallazgo que también se corroboró en el estudio de Hill (1945), en el de McGinnis (1958), en el Hudson y Henze (1969) y en el de Buss (1989) que lo replica con 1491 estadounidenses a mediados de los 80 .

El valor que las féminas otorgan a los recursos económicos se ha puesto de manifiesto en otros contextos Kenrick, Sadalla, Groth y Trost (1990) diseñaron un método para descubrir cuántas personas valoran distintos atributos en un cónyuge. Para ello pedían a hombres y mujeres que indicaran en percentiles mínimos de todas las características que les parecieran aceptables. Las mujeres estadounidenses universitarias indicaron que su percentil mínimo aceptable para un marido, con respecto a su capacidad de ganar dinero, es el percentil 70, por encima del $70 \%$ del resto de los hombres. El mínimo masculino aceptable con respecto a la capacidad de la esposa de ganar dinero solo fue de 40 .

Los resultados obtenidos en nuestro estudio, como en otros mencionados con anterioridad, muestran que las mujeres de todas las culturas presentes y pasadas, escogen a los hombres por su aparente capacidad de acumular futuros recursos, basándose en determinadas características de personalidad.

Respecto a las otras características valoradas en la pareja tanto en nuestro estudio como en el trabajo de las 18 características evaluadas en el estudio sobre las 37 culturas de Buss (1989) sobre la elección de la pareja, la segunda y tercera más valoradas después del amor son el carácter formal. Así los rasgos más apreciados en la pareja cuando es estable son la amabilidad, la comprensión la inteligencia. Las expectativas son idénticas para las características como la sinceridad, prevaleciendo los rasgos afectivos sobre los de carácter físico, que es donde se obtienen diferencias estadísticamente significativas en las preferencias entre mujeres y hombres.

La sinceridad indica que la pareja es capaz de comprometerse. Los recursos, el compromiso y la protección, junto con el valor que las mujeres conceden a la salud masculina, asegura que los hombres podrán aportar tales beneficios durante un largo tiempo. Para que un emparejamiento a largo plazo tenga éxito requiere una alianza cooperativa prolongada con otra persona para conseguir objetivos mutuamente beneficiosos. Esto requiere compatibilidad que no es otra cosa que buscar lo similar en la pareja. Así los hombres y las mujeres que tienen muchas características similares tienden a emparejarse. $\mathrm{La}$ tendencia hacia personas parecidas se manifiesta en los valores, la inteligencia y la pertenencia al grupo. Se busca como pareja a quien tiene valores políticos y sociales similares, inteligencia similar y por tanto existe semejanza en las características de la personalidad. Buss en 1994 publica un estudio sobre parejas de novios en Cambridge (Massachusetts), mide la personalidad y el nivel de inteligencia de 108 sujetos que mantenían una relación de pareja. Las mediciones se realizaron por separado ya que tenían que manifestar, para las mismas cualidades, sus preferencias en la pareja ideal. Los resultados mostraron que las mujeres tenían preferencia por parejas que son similares a ellas en muchos aspectos, como ser valiente, dominante, activo, afectuoso, agradable y amable, responsable y especialmente inteligente, perceptivo y creativo.

En nuestro estudio de los universitarios, las preferencias por la inteligencia son seleccionadas con más frecuencia por los hombres, tanto cuando se les cuestiona en una pareja estable, como cuando se les pregunta por las características que debería tener la pareja en términos más genéricos. Ambos sexos coinciden en seleccionar y priorizar otros valores personales como la sinceridad, simpatía, amabilidad y la capacidad de iniciativa. Todas ellas actitudes de garantía y estabilidad frente al esfuerzo de la procreación y las necesidades de atención en la crianza.

\section{Preferencias en los emparejamientos ocasionales/fugaces}

Schmith y Buss (1996) llevaron a cabo un estudio sobre emparejamientos temporales y permanentes para descubrir qué características se valoraban en un posible cónyuge frente a las valoradas en un 
compañero sexual ocasional. El estudio lo realizaron entre los estudiantes de la Universidad de Michigan. Cientos de personas valoraron 67 características con respecto a si eran o no deseables en la pareja a largo o a corto plazo. Las mujeres juzgaron muy deseable en una posible pareja estable la probabilidad de éxito en su profesión y la posesión de una carrera prometedora. Estas características eran más deseables en el cónyuge que en un compañero sexual ocasional. Estas diferencias las constatan los estudios de Bleske-Rechek y Buss (2006) y los de Schmitt y Buss (2001), que exploran la perspectiva romántica de la atracción sexual.

Nuestro estudio muestra que el atractivo físico es la característica más deseada en el emparejamiento ocasional, después la simpatía y la amabilidad, y que existen diferencias estadísticamente significativas entre lo que buscan hombres y mujeres. Las féminas valoran más la simpatía y la posición social y los hombres, el atractivo físico y la personalidad enigmática en las relaciones fugaces. Así, los resultados obtenidos en nuestro estudio mantienen los estereotipos sobre la importancia que las mujeres dan a la posición social (indicadora de si se poseen o no recursos) y el que los hombres aprecian más el atractivo físico (indicador de juventud, salud y fertilidad).

En suma las cualidades que las personas prefieren no son estáticas. Como las características cambian, quienes buscan pareja deben evaluar su potencial futuro. Evaluar el valor de un hombre como compañero implica ver más allá de su posición actual y examinar su potencial. Cada atributo por separado constituye un componente del valor que tiene un hombre, como pareja, para una mujer y viceversa. Cada una de sus preferencias no resuelven completamente el problema de elegir pareja. El valor de un posible compañero depende de la perspectiva individualizada, personalizada y contextualizada de quien realiza la elección.

Quizá somos tan selectivos porque las relaciones de pareja proporcionan una de las satisfacciones más profundas de la vida, pero también el emparejamiento genera conflictos, competencia y manipulación.

\section{El modelo de pareja de los progenitores y su influencia}

Por último, los resultados sobre la influencia de los progenitores en el modelo de pareja muestran que más de la mitad de los universitarios piensan que sí les ha influido el modelo, frente a menos de una tercera parte que lo niega. Pero lo realmente curioso es que sean las mujeres las que más responden afirmativamente. Quizás las mujeres se interesan más por el tipo de relaciones de pareja, reflexionan más sobre ello y lo comentan en sus núcleos de amistad, y todo ello las lleva a ser más conscientes $\mathrm{y} / \mathrm{u}$ observadoras sobre una posible influencia del modelo de sus progenitores.

Son sorprendentes los resultados obtenidos en la selección de las características que más agradan del padre y de la madre, los perfiles de respuesta diferenciados entre padre y madre. Así, al padre se le valora más la inteligencia, la comprensión y la amabilidad, destacando que los varones seleccionan más la sinceridad y la comprensión y las mujeres la personalidad enigmática. En cambio, a la madre se le valora primero la comprensión, después la amabilidad y la sinceridad y sólo se obtienen diferencias en las respuestas que dan hombres y mujeres respecto a la fidelidad que es más valorada por las mujeres.

\section{Referencias}

Betzig, L. (1986). Despotism and differential reproduction: A Darwinian view of history. Howtorne, New York: Aldine de Gruyter.

Bleske-Rechek, A. \& Buss, M. (2006). Sexual strategies pursued and mate attraction tactics deployed. Personality and Individual Differences 40, 1299-1311.

Buss, D. M. (1984). Toward a psychology of person-environment (PE) correlation: The role of spouse selection. Journal of Personality and Social Psychology, 47, 361-377.

Buss, D. M. (1985). Human mate selection. American Scientist, 73, 47-51.

Buss, D. M. (1989). Sex differences in human mate preferences: Evolutionary hypotheses tested in 37 cultures. Behavioural and Brain Sciences, 12, 1-49.

Buss, D. M. (1994). The evolution of Desire. New York: Harper Collins Publishers.

Buss, D. M., Abbott, M., Angleitner, A., Asherian, A., Biaggio, A., Blanco-Villaseñor, A., Bruchon-Schweitzer, M., Ch'U, Hai-Yuan, Czapinski, J., De Raad, B., Ekeham-mar, B., Fioravanti, M., Georgas, J., Gjerde, P., Gutman, R., Hazan, F., Iwawaki, S., Janakiramaiah, N., Khosroshani, F., Kreitler, S., Lachenicht, L., Lee, M., Liik, K., Little, B., Lohamy, N., Makim, S., Mika, S., Moadel-Shahid, M., Moane, G., Montero, M., Mundy-Castle, A. C., Lttle, B., Niit, T., Nsenduluka, E., Peltzer, K., Pienkwski, R., Pirtila-Backman, A., Ponce De León, J., Rousseau, J., Runco, M. A., Safir, M. P., Samuels, C., Sanitioso, R., Schweitzer, B., Serpell, R., Smid, N., Spencer, C., Tadinac, M., Todorova, E. N., Troland, K., Van Den Brande, L., Van Heck, G., Van Langenhove, L. \& Yang, K.-S. (1990). International preferences in selecting mates: A study of 37 cultures. Journal of cross-cultural psychology, 21, 5-47.

Buss, D. M. \& Barnes, M. F. (1986). Preferences in human mate selection. Journal of Personality and Social Psychology, 50, 559-570.

Buss, D. M. \& Schmitt, D. P. (1993). Sexual strategies theory: An evolutionary perspective on human mating. Psychological Review, 100, 204-232. 
Demoscopia (1997). El País, 23/04/1997.

Haberman, S. J. (1978). Analysis of qualitative data (Vol I). New York: Academic.

Harrison, A. A \& Saeed, L. (1977). Let's make a deal: An analysis of revelations and stipulations in lonely heart's advertisements. Journal of Personality and Social Psychology, 35, 257-264.

Hill, R. (1945). Campus values in mate selection. Journal of Home Economics, 37, 554-558.

Hudson, J. W. \& Henze, L. F. (1969). Campus values in mate selection: A replication. Journal of Marriage and the family, 31, 772-775.

Ford, C. \& Beach, F. (1951). Patterns of sexual behaviour. New York: Harper \& Row.

Kernick, D. T., Sadalla, E. K., Groth, G. \& Trost, M. R. (1990). Evolution, traits, and stages of human courtship: Qualifying the parental investment model. Journal of Personality, 58, 97-116.

Langhorne, M. C. \& Secord, P. F. (1955). Variations in marital needs with age, sex, marital status, and regional composition. Journal of Social Psychology, 41, 19-37.

Laumann, E., Gagnon, J., Michael, R., Michaels, S. (1994). The social organization of sexuality. Chicago, EEUU: Chicago University press.
Maté C. \& Acarín, N. (2010) Encuesta sobre las relaciones sexuales a estudiantes de la UPF (20 a 27 años) (en prensa). McGinnis, R. (1958). Campus values in mate selection. Social Forces, 35, 368-373.

Singh, D. (1993). Adaptative significance of waist-to-hip ratio and female physical attractiveness. Journal of Personality and Social Psychology, 65, 293-307.

Schmith, D. P. \& Buss, D. M. (1996). Strategic self-promotion and competitor derogation: sex and context effects on the perceived effectiveness of mate attraction tactics. Journal of Personality, 70, 1185-1204.

Schmith, D. P. \& Buss, D. M. (2001). Human mate poaching: Tactics and Temptations for infiltrating existing mateships. Journal of Personality and Social Psychology, 80, 6, 894-917.

Suggs, D. \& Miracle, A. (Eds). (1993). Culture and human Sexuality. Pacific Groves, CA: Brooks/Cole.

Wiederman, M. W. (1996). Evolved gender differences in mate preferences: Evidence from personal advertisements. Ethology and Sociobiology, 14, 5, 331-351.

Wiederman, M. W. \& Allgeier, E. (1992). Gender differences in mate selection criteria: Sociobiological or socioeconomic explanations? Ethology and Sociobiology, 13, 2, 115-124. 